\title{
Modulating Effect of Vitamin D on iNOS, PCNA and $\alpha$-SMA Expression Against Diclofenac Sodium Induced Gastric Injury in Rats
}

\author{
Sahar Youssef* \\ Anatomy Department, Faculty of Medicine for Girls, Al-Azhar University, Cairo, Egypt \\ *Corresponding author. E-mail: sahar_sayed@yahoo.com
}

Received date: Dec 21, 2019; Revised date: Apr 20, 2020; Accepted date: May 5, 2020

\section{Abstract}

B ACKGROUND: Diclofenac sodium is a nonsteroidal anti-inflammatory prescription, widely used in the management of many inflammatory diseases but the side effects limiting its clinical use. The present work was carried out to detect the ameliorative effect of vitamin $\mathrm{D}$ against diclofenac sodium induced gastric injury in adult male albino rats.

METHODS: Forty adult male Wistar albino rats were classified into four groups: G-I received no treatment (control group), G-II orally received $500 \mathrm{IU} / \mathrm{kg}$ of vitamin D daily, G-III intraperitoneally received $3 \mathrm{mg} / \mathrm{kg}$ of diclofenac sodium daily, and G-IV received both $500 \mathrm{IU} /$ $\mathrm{kg}$ of vitamin D and $3 \mathrm{mg} / \mathrm{kg}$ of diclofenac sodium daily for 14 days. Specimens from rats' stomach were processed for light microscopy. Immunohistochemical examination was carried out to detect inducible nitric oxide synthase (iNOS), proliferating cell nuclear antigen (PCNA), and alpha smooth muscle actin ( $\alpha$-SMA). The morphometric results were analyzed statistically.
RESULTS: Gastric sections of G-III displayed inflammatory cellular infiltrations and dilated congested blood vessels. Some of the gastric gland cells showed cytoplasmic vacuolation, dilated gastric pits, and cystic dilatation. There was a significant increased Masson's trichrome stain and a significant decrease in PAS. The mean area percentage of iNOS and $\alpha$-SMA expression showed a statistically significant increase. The PCNA positive cells were significantly decreased in the isthmus and neck region compared with the control. While in contrast, G-IV prevented the gastric injury by increasing PAS and PCNA but decreasing Masson's trichrome stain, iNOS and a-SMA expression.

CONCLUSION: Vitamin D administration prevented the structural alterations of the gastric rat induced by diclofenac sodium.

KEYWORDS: diclofenac sodium, $\alpha$-SMA, iNOS, PCNA, vitamin D

Indones Biomed J. 2020; 12(2): 165-76

\section{Introduction}

Non-steroidal anti-inflammatory drugs (NSAIDs) are prescribed for its analgesic, antipyretic, and antiinflammatory possessions. They are commonly used to treat many inflammatory diseases due to their role in relieving of pain, decreasing inflammation and fever. The long period using NSAIDs is frequently related to gastric mucosal ulcer and leads to serious complications such as bleeding and perforation. In humans, NSAIDs is associated with adverse effects such as gastrointestinal injury, renal dysfuction, and cardiovascular damage. (1) Prostaglandins are formed from arachidonic acid by two isoforms of cyclooxygenase (COX) that defend the gastric mucosa against injury. However, NSAIDs act by blocking of COX enzymes and inhibiting the production of prostaglandins. Others have been reported their action is due to neutrophil activation.(2) Also, acid in the lumen of the stomach contributed to the mechanism of NSAIDs induced ulcers and bleeding by damaging the 
regeneration procedure interfering with haemostasis and deactivating numerous growth factors that are essential in the mucosal protection and repair.(3) Mechanisms of damage to the stomach involve the oxygen free radicals. (4) Diclofenac sodium is one of the NSAIDs widely used as an anti-inflammatory and analgesic drug derived from phenyl acetic acid. Its action is completed by decreasing the synthesis of prostaglandins through prevention of COX enzymes mainly COX2.(5) On the other hand, gastric ulcer diseases have been reported after chronic administration of diclofenac for rheumatoid and osteoarthritis patients. (6) To get safe to use diclofenac sodium requires a better understanding of the pathogenesis of diclofenac sodium induced gastric ulcer disease. Previous reports suggested that most important protective factors for mucosa are adequate blood flow, motility, cell regeneration, bicarbonate, mucus, prostaglandins and nitric oxide.(7) Nitric oxide produced from stimulation of inducible nitric oxide synthase (iNOS) action in the gastric ulcer induction through the formation of reactive oxygen species, a powerful cytotoxic oxidant that causes gastric injury.(8) Both proliferation and apoptosis processes are in balance however if this balance is disrupted either due to increase the apoptosis and/or decrease the proliferation, mucosal damage will occur. Proliferating cell nuclear antigen (PCNA) is a marker for cell proliferation that extent its extreme level in the nucleus during late G1/S phase of the cell cycle and this specifies the onset of DNA synthesis.(9)

Ulcer closure is a combination process of regional epithelial migration, proliferation and myofibroblast contraction. Myofibroblasts appeared at the site of injury, secrete the extracellular matrix proteins that support contraction of granulation tissue through the expression of alpha-smooth muscle actin ( $\alpha$-SMA) which plays an important role in fibrogenesis.(10)

Gastric ulcer management is carried out using antacid prescriptions; however, most of these medications have severe side effects. Currently, studies directed towards the use of safe products and reduced diclofenac adverse effect by the appropriate use of antioxidants.

Vitamin D is a potent antioxidant. It is a fat soluble vitamin and plays an important role in regulation of bone metabolism and stimulates calcium absorption in the gut. Vitamin D has other roles in the body including cellular differentiation, proliferation, and immune function.(11) Vitamin D is a steroid hormone that obtained from food and sun exposure. Additional functions of the vitamin D include anti-oxidative, anti-inflammatory and anti-fibrotic effects.(12) The above mentioned possessions of vitamin D encouraged us to examine the ameliorative potential of this vitamin against the gastric alterations induced by diclofenac sodium in male albino rats.

Thus, the current study was designed to evaluate the effect of diclofenac sodium on the fundic region of stomach adult male albino rat and to elucidate the fundamental mechanisms of vitamin D supplementation using the immunohistochemical investigation of iNOS, PCNA and $\alpha$-SMA.

\section{Methods}

\section{Animals}

Forty male albino Wistar rats, with a body weight ranged from $200 \pm 20 \mathrm{~g}$ were used in the present study. Rats were purchased from the animal house of Faculty of Medicine, Assiut University, Egypt. Rats were well kept under harmless sterile conditions for acclimatization for two weeks before starting the experiment. They were housed in stainless steel cages at $24 \pm 1^{\circ} \mathrm{C}, 12 \mathrm{~h}$ dark/light cycles. They were allowed standard commercial pellets for feeding, and free access to water ad libitum. All animal processes were in agreement with the guidelines of the experimental animals and coincide with national Institute of Health protocol and through international guidelines.

\section{Experimental Design}

The rats were divided into four groups, with 10 rats in each group. G-I was the control group that received no treatment. G-II was the vitamin D group that orally received vitamin D in a dose of $500 \mathrm{IU} / \mathrm{kg}$ daily for 14 days. Vitamin D (cholecalciferol) in the form of oral drops $2800 \mathrm{IU} / \mathrm{mL}$ was obtained from Vidrop, Medical Union Pharmaceuticals, Abu-Sultan, Egypt. G-III was the diclofenac sodium group that intraperitoneally received 3 $\mathrm{mg} / \mathrm{kg}$ of diclofenac sodium daily for 14 days. Diclofenac sodium (voltaren) ampoules containing $75 \mathrm{mg} / 3 \mathrm{~mL}$ was produced by Novartis Pharma company (Basel, Switzerland). Lastly, G-IV was the group that receive both vitamin D and diclofenac sodium in similar doses of G-II and G-III for 14 successive days. $(13,14)$

\section{Histological Analysis}

The abdomen of the rats was opened and stomach specimens were fixed in $10 \%$ neutral buffered formalin and accomplished for histological analysis. Paraffin blocks were sectioned into $5 \mu \mathrm{m}$ thickness and stained with Hematoxylin and Eosin (H\&E) for general examination (15), Masson's 
trichrome was used to detect collagen fibers and Periodic acid Schiff (PAS) to detect mucosal glycoprotein (16).

\section{Immunohistochemical Analysis}

Immunohistochemical staining for iNOS, PCNA and $\alpha$-SMA was conducted using the avidin-biotin peroxidase technique according to previous studies method.(17,18) Three $\mu \mathrm{m}$ thick sections were deparaffinized in xylene and then rehydrated in alcohol. Methanol comprising of $0.03 \%$ hydrogen peroxide was carried out for 20 minutes to remove the endogenous peroxidase activity. To block nonspecific antibody, stomach sections were incubated for 20 minutes with normal serum. The sections were incubated with antiiNOS rabbit polyclonal antibody (1:200 diluted, Abcam, Cambridge, UK). Successively, sections were incubated with biotinylated goat anti-rabbit IgG secondary antibody (1:100 diluted, Abcam). Other sets of slides were incubated with a primary antibody (PCNA, mouse monoclonal antibody, PC10) (1:200 diluted, Thermo Fischer Scientific, Waltham, Massachusetts, USA). Consequently, the biotinylated goat anti-mouse IgG secondary antibody (1:200 diluted, Abcam) was carried out to detect the primary antibody to PCNA. Others were incubated with $\alpha$-SMA mouse monoclonal antibody (1:100 diluted, DAKO, Glostrup, Denmark). Anti-mouse IgG (1:500 diluted, Sigma-Aldrich, St. Louis, Missouri, USA) was used as the secondary antibody. All stomach sections were successively stained with biotinylated IgG for 10 minutes and avidin biotin horseradish peroxidase complex for 15 minutes. Visualization of the reaction was conducted by adding the chromogen diaminobenzidine (DAB) (Dako) to slides. Slides were washed with distilled water and sections were counterstained with Mayer's hematoxylin. Stomach sections were dehydrated, reduced transparent with xylene, and cover slipped. Normal stomach tissues were used as positive controls. The negative controls were treated without the primary antibody. The photography was conducted at the Mycology and Biotechnology Unit, Al-Azhar University, Cairo, Egypt.

\section{Morphometric Analysis}

The Leica Qwin 500 program, Image analyzer computer system (Leica, Cambridge, UK) was used. To estimate the mucosal height $(\mu \mathrm{m})$, the perpendicular distance between the stomach mucosal surface and the muscularis mucosa were measured using the same H\&E stained sections that used for histological study. Ten evaluations were considered in five non-overlapping fields at magnification of x100. Also, the area \% of the collagen content using Masson's trichrome stained sections, area $\%$ of PAS positive reaction in PAS-stained sections, area $\%$ of positive immune reaction for iNOS, $\alpha$-SMA were measured. The image analyzer was calibrated to transform the measurement units (pixels) formed by the image analyzer program into the definite micrometer units. Using measuring field menu, then the area percentage and the standard measuring frame were chosen. Stomach section was enclosed inside the standard measuring frame and then the area were masked by a blue binary colour to be evaluated. $(17,18)$ These measurements were conducted using an objective lens of magnification $\mathrm{x} 40$ and ten reading were calculated in five non-overlapping fields from each rat. The mean number of PCNA positive cells in the immunostained slides was assessed. Positive PCNA staining was calculated based on the formulation: the level of positivity $=$ Number of positive cells / number of counted cells multiplied by 100.(19)

\section{Data Analysis}

Data analysis was carried out using SPSS software (version 16, Chicago, Illinois, USA). Results obtained from the morphometric analysis were expressed as means \pm standard deviation. One-way analysis of variance (ANOVA) followed by Tukey's post hoc test to conclude the differences among the mean values of different groups. A probability of $p<0.05$ was considered statistically significant.

\section{Results}

\section{Hematoxylin and Eosin (H\&E)}

H\&E stained sections of G-I and G-II showed that normal architecture of fundic glands, consists of isthmus, neck and base areas opened into the surface by short narrow gastric pits (Figure 1A, 1B). The upper part of lining epithelium formed of simple columnar mucous secreting cells with foamy apical cytoplasm and basal oval nuclei. The isthmus was lined by surface mucous secreting cells (Figure 2A, 2B). The middle neck region showed large parietal cells with central rounded nuclei and eosinophilic cytoplasm and surface mucous neck cells. The majority of chief cells occupied at the base with basal oval nuclei, basal basophilic cytoplasm, and pale apical granular part (Figure 2A,2B).

H\&E stained sections of G-III revealed variable degrees of mucosal lesions. Some sections showed marked loss of normal architecture (Figure 1C), widening of gastric pits (Figure 2E, 2F), congested dilated blood vessels in between the fundic glands and in the submucosa (Figure $1 \mathrm{C}, 2 \mathrm{G})$. Some chief and parietal cells appeared vacuolated (Figure 2C). Focal areas of extensive mononuclear cellular 

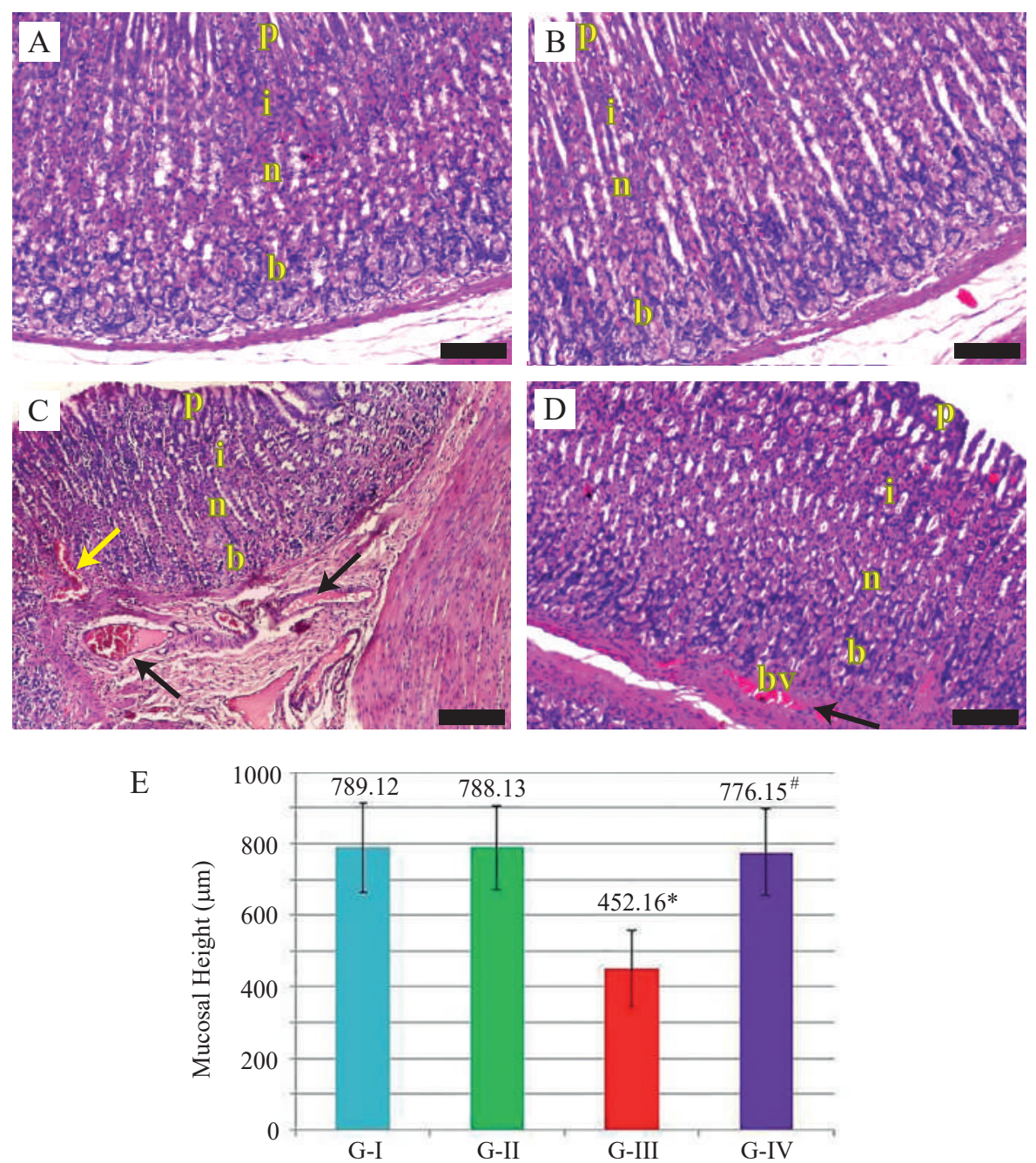

Figure 1. Photomicrographs of stomach sections of different studied groups. A: G-I, showing regular arrangement of fundic mucosa glands that open into the lumen by short narrow gastric pits. B: G-II, showing normal histological architecture of the fundic glands and the lining cells. C: G-III, showing the disorganized gastric mucosa which consists of wide gastric pits, isthmus, neck and base. Numerous engorged blood vessels (black arrows) in the submucosa and in between the basal part of glands (yellow arrow). D: G-IV, showing restoration of the normal structure of the gastric mucosa with variable gastric pits. Mildly congested blood vessels and few inflammatory cellular infiltrations (black arrow) can be seen. Black bar: $200 \mu \mathrm{m}$; p: gastric pits; i: isthmus; n: neck; b: base; bv: blood vessels. E: Graph bars represent mucosal height detected in the different studied groups. *Significant decrease of G-III vs. G-I, while ${ }^{\#}$ significant increase of G-IV vs. G-III at $p<0.001$.

infiltrations (Figure 2D), consistent appearance of distorted, disorganized, dilated glands and the glandular cystic dilatations lined by flattened epithelial cells (Figure 2C, 2E).

H\&E stained sections of G-IV revealed more or less intact mucosa with short narrow pits (Figure1 D), however, some glandular cells still had vacuolated cytoplasm (Figure $2 \mathrm{H})$. The mucosal height $(\mu \mathrm{m})$ was statistically significant decreased in the G-III (452.16 \pm 107.12$)$ as compared to the control group, however, it was significantly increasing in the G-IV (776.15 \pm 121.25$)$ as compared to G-III. No statistically significant difference between G-I (789.12 \pm 125.23$)$ and G-II (788.13 \pm 117.14$)$ (Figure 1E).

\section{Masson's Trichrome}

Sections of G-I and G-II revealed few collagen fibers in between the basal parts of fundic glands and in submucosa (Figure 3A, 3B). G-III showed dense irregularly arranged collagen fibers between the distorted fundic glands and around dilated congested blood vessels in the submucosa (Figure 3C). G-IV showed decrease the collagen fibers in between fundic glands and in the submucosa (Figure 3D). The mean area percentage of collagen fibers was significantly increasing in G-III $(9.65 \pm 1.22)$ as compared to G-I. However, it was significantly decreasing in G-IV (5.14 \pm 0.55$)$ as compared to G-III. No significant difference between G-I (4.32 \pm 0.22$)$ and G-II $(4.34+0.65)$ (Figure 3E). 

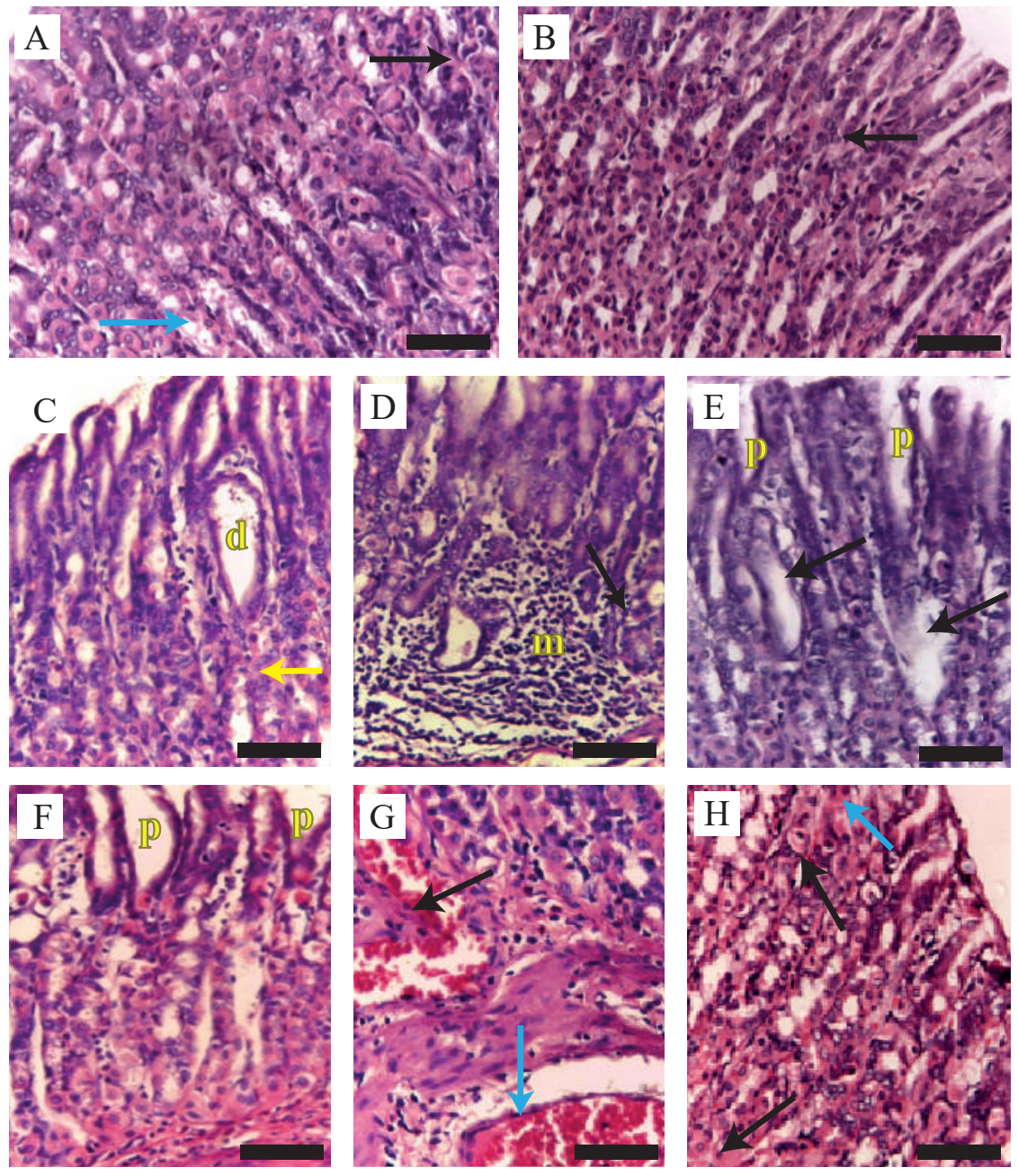

Figure 2. Photomicrographs of gastric mucosal sections from different studied groups. A: G-I, showing regularly arranged fundic glands with short gastric pits. Normal architecture of long, straight and packed gastric glands, intact parietal cells (black arrow) and chief cells (blue arrow). B: G-II, showing normal architecture of fundic glands with narrow gastric pits, normal appearance of mucous surface, parietal cells (black arrow) and chief cells (blue arrow). C: G-III, showing a glandular cystic dilatation and marked vacuolization of the cytoplasm of some parietal cells (yellow arrow). D: G-III, showing disordered fundic glands (black arrow) with excessive mononuclear cells infiltration and aggregation. E: G-III, showing some glands are disorganized and dilated (black arrows) and wide gastric pits. F: G-III, showing disordered widening gastric pits. G: G-III, showing dilated congested blood vessels between widely separated fundic mucosal glands (black arrow) and in the submucosa (blue arrow). H: G-IV, showing some parietal cells with mild vacuolation (blue arrow). Some intact parietal cells (black arrows) and intact chief cells. Black car: $50 \mu \mathrm{m}$; p: gastric pits, d: cystic dilatation; m: mononuclear cells.

\section{Periodic Acid Schiff (PAS)}

Sections of G-I and G-II showed intense PAS positive reaction on the thick mucus film over the surface epithelium and spread down to the gastric pits, isthmus and neck areas (Figure 4A, 4B). Sections of G-III revealed an interrupted mucosal layer and weak PAS positive reaction on the mucosal surface, pits and isthmus regions but negative PAS reaction in the neck region and no mucus film were observed (Figure 4C). Sections of G-IV showed intense PAS positive reactivity on the thin mucous film over the surface epithelium spreading down into the gastric pits; isthmus and neck (Figure 4D). The mean area percentage in the PAS sections was significantly decreasing in G-III $(6.6 \pm 0.265)$ as compared to G-I. However, the mean area was significantly increasing in G-IV (13.45 \pm 0.22$)$ as compared to G-III. No statistically significant difference between G-I $(14.6 \pm 0.76)$

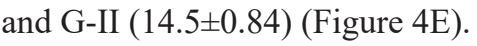

\section{iNOS Expression}

iNOS-immunohistochemical stained sections from G-I, G-II revealed a faint iNOS immunohistochemical positive cytoplasmic reaction in the form of a brownish coloration mainly in the basal regions (Figure 5A, 5B). While, sections obtained from G-III revealed a strong iNOS on the surface epithelium, gastric pits, the isthmus and moderate in the basal areas (Figure 5C). Sections from G-IV showed a 

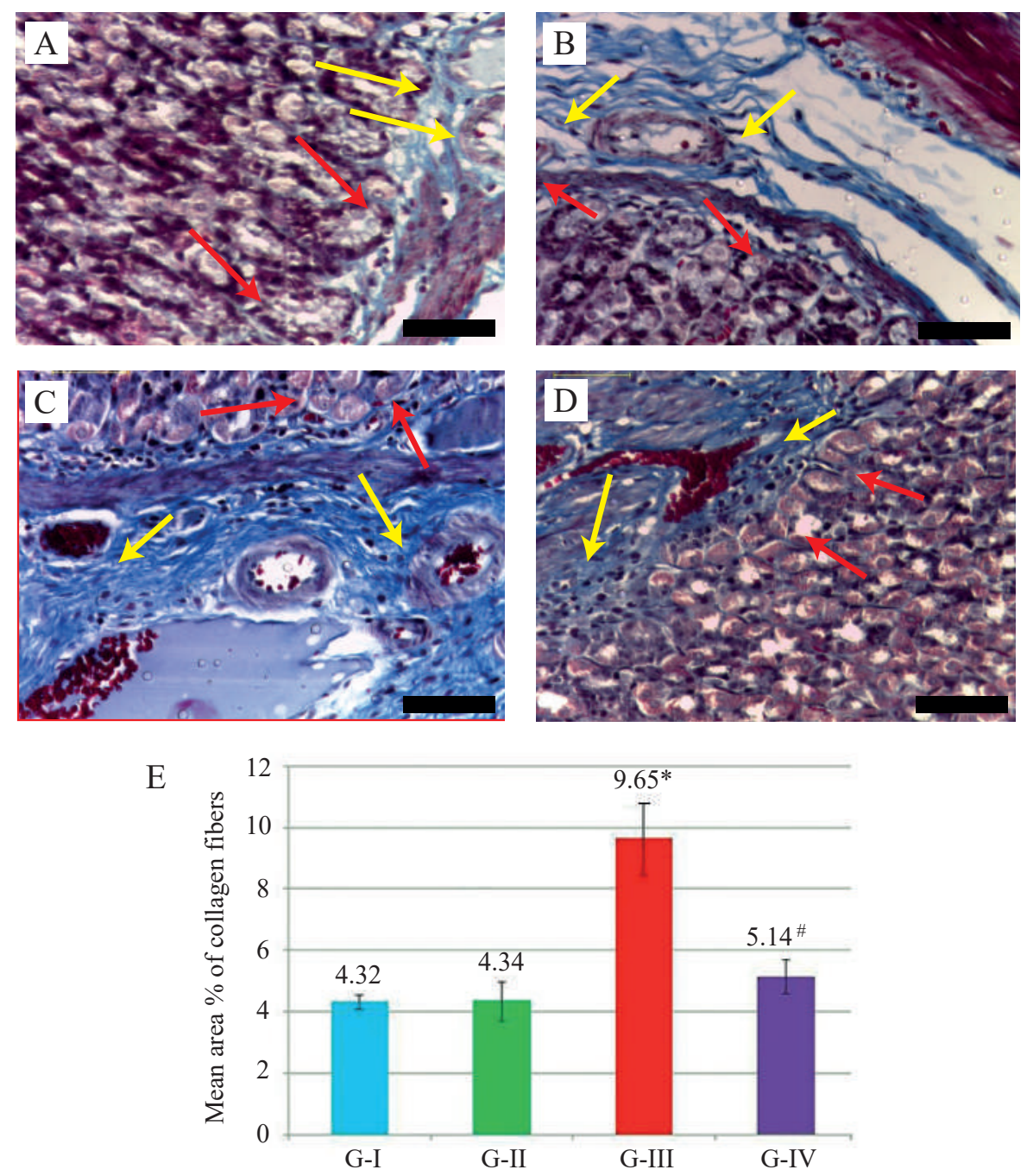

Figure 3. Photomicrographs of fundic gastric sections from different studied groups. A: G-I, showing few collagen fibers between the fundic glands in the basal parts (red arrows) and in the submucosa (yellow arrows) which contains blood vessels. B: G-II, showing little collagen fibers in the basal parts (red arrows) of fundic glands and around blood vessels (yellow arrows) in the submucosa. C: G-III, showing marked increase in the deposition of the collagen fibers in between the basal parts of the gland (red arrows) and around dilated congested blood vessels in the submucosa (yellow arrows). D: G-IV, showing moderate decrease in collagen fibers between the fundic glands especially at the base (red arrows) and in submucosa (yellow arrows). Black bar: $50 \mu \mathrm{m}$. E: Graph bars represent area percentage of collagen fibers detected in the different studied groups. *Significant increase of G-III vs. G-I, while *significant decrease of G-IV vs. G-III at $p<0.001$.

moderate iNOS reaction in the basal region (Figure 5D). The mean area percentage of iNOS of the fundic mucosa was significantly increasing in G-III ( $30.76 \pm 1.55)$ as compared to G-I. However, iNOS was statistically significant decreased in G-IV ( $8.99 \pm 0.88)$ as compared to G-III. No statistically significant difference was found between G-I $(6.13 \pm 0.50)$ and G-II (6.12 \pm 0.33$)$ (Figure 5E).

\section{PCNA Expression}

PCNA immunohistochemical stained sections showed dark brown cytoplasmic granules at the isthmus and middle zone of gastric glands of G-I and G-II (Figure 6A, 6B). The PCNA positive cells were decreased in G-III in the isthmus and middle zone (Figure 6C) as compared to G-I. Numerous immunopositive PCNA stained cells were detected in sections from G-IV in the isthmus and neck cells down to basal parts of fundic glands (Figure 6D). The mean number of PCNA positive cells was significantly decreased in G-III (82.7 \pm 2.67$)$ as compared to G-I, however, it was significantly increased in G-IV (121 \pm 3.3$)$ in comparison to G-III. No statistically significant difference between G-I $(95.2 \pm 1.8)$ and G-II $(95.3+1.9)$ (Figure 6E).

\section{a-SMA Expression}

$\alpha$-SMA immunohistochemical stained sections from G-I and G-II revealed brownish immunohistochemical reaction 

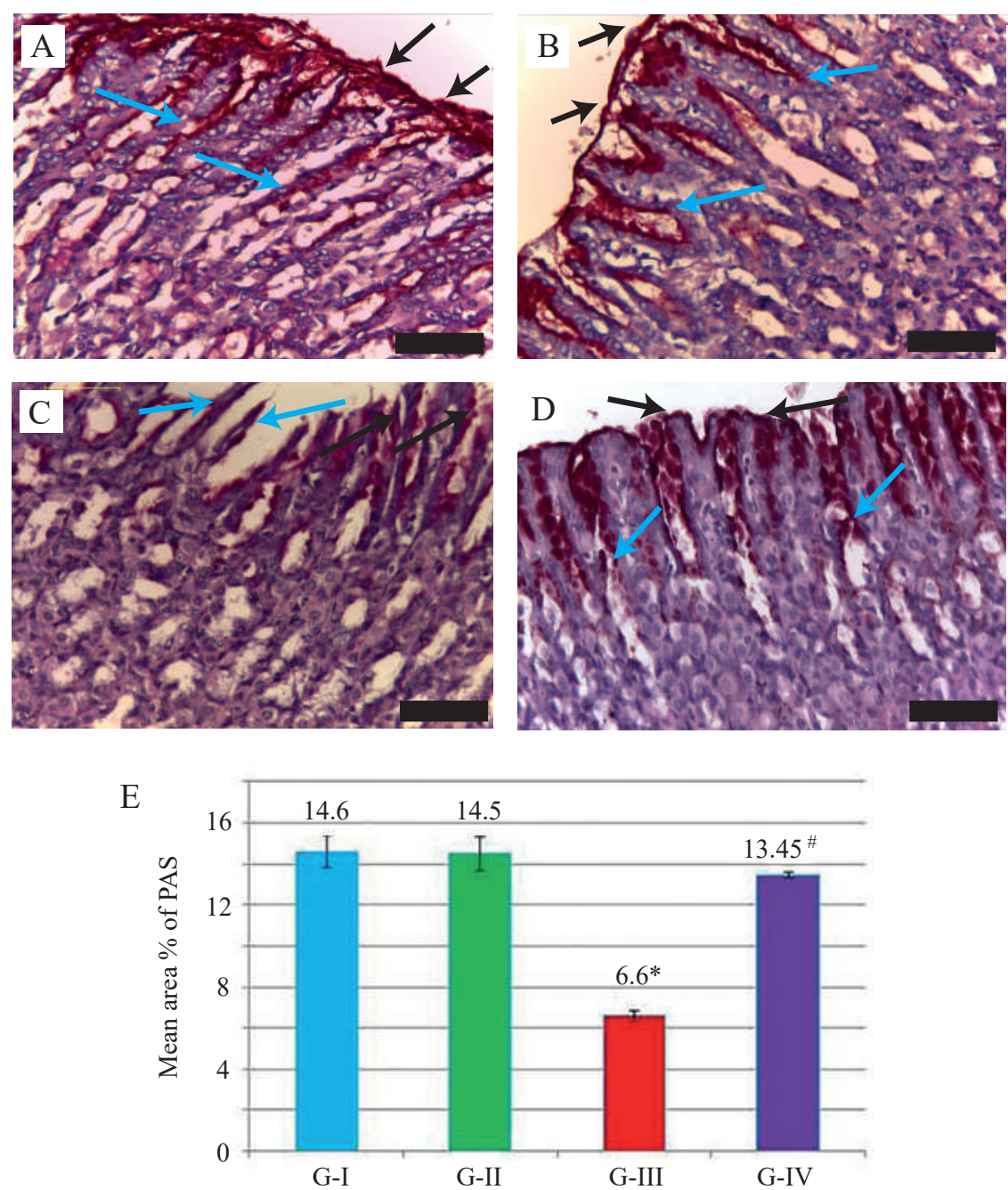

Figure 4. Photomicrographs sections of fundic stomach mucosa of different studied groups. A: G-I, showing strong PAS positive reaction covering the surface mucous cells (black arrows) and extending to the gastric pits and isthmus of glands (blue arrows). B: G-II, showing localized strong PAS positive reaction in the surface mucous coat (black arrows) and in the isthmus (blue arrows). C: G-III, showing faint and interrupted PAS positive material of the surface mucous coat and extend to fill gastric pits (black arrows) with areas of focal loss (blue arrows). D: G-IV, showing increased PAS positive material covering the surface columnar cells (black arrows) and extending to the pits and isthmus (blue arrows). Black bar: $50 \mu \mathrm{m}$. E: Graph bars represent area percentage of PAS reaction in the different studied groups. *Significant decrease of G-III vs. G-I, while "significant increase of G-IV vs. G-III at $p<0.001$.

mainly in the muscularis mucosa, muscularis externa and around blood vessels (Figure 7A, 7B). Sections obtained from G-III revealed a strong $\alpha$-SMA immunohistochemical staining in the muscularis mucosa, muscularis externa and in between the basal parts of mucosal glands (Figure 7C). Sections from G-IV showed positive $\alpha$-SMA immunohistochemical reaction in muscularis mucosa, muscularis externa and few positive staining in between basal parts of fundic mucosal glands (Figure 7D). The mean area percentage of $\alpha$-SMA was significantly increased in G-III $(55.39 \pm 2.03)$ as compared to G-I. However, $\alpha$-SMA was significantly decreased in G-IV $(32.17 \pm 1.14)$ as compared to G-III. No significant difference was found between G-I (25.37- \pm 1.07$)$ and G-II (25.39- \pm 1.09$)$ (Figure7E).

\section{Discussion}

In this study, the histological findings suggested that there was toxicity of the gastric mucosa upon administration of diclofenac sodium. This was shown by the appearance of cellular infiltration in in the diclofenac sodium group. This could be attributed due to damage of integrity of the intercellular junctions. Data from the literature have confirmed experimentally that after exposure to NSAIDs, gastric mucosa exposed to the effect of acid and enzymes, and later bacterial invasion.Then, bacteria secrete the chemotactic factors such as macrophage, lymphocytes and neutrophils.(20) 

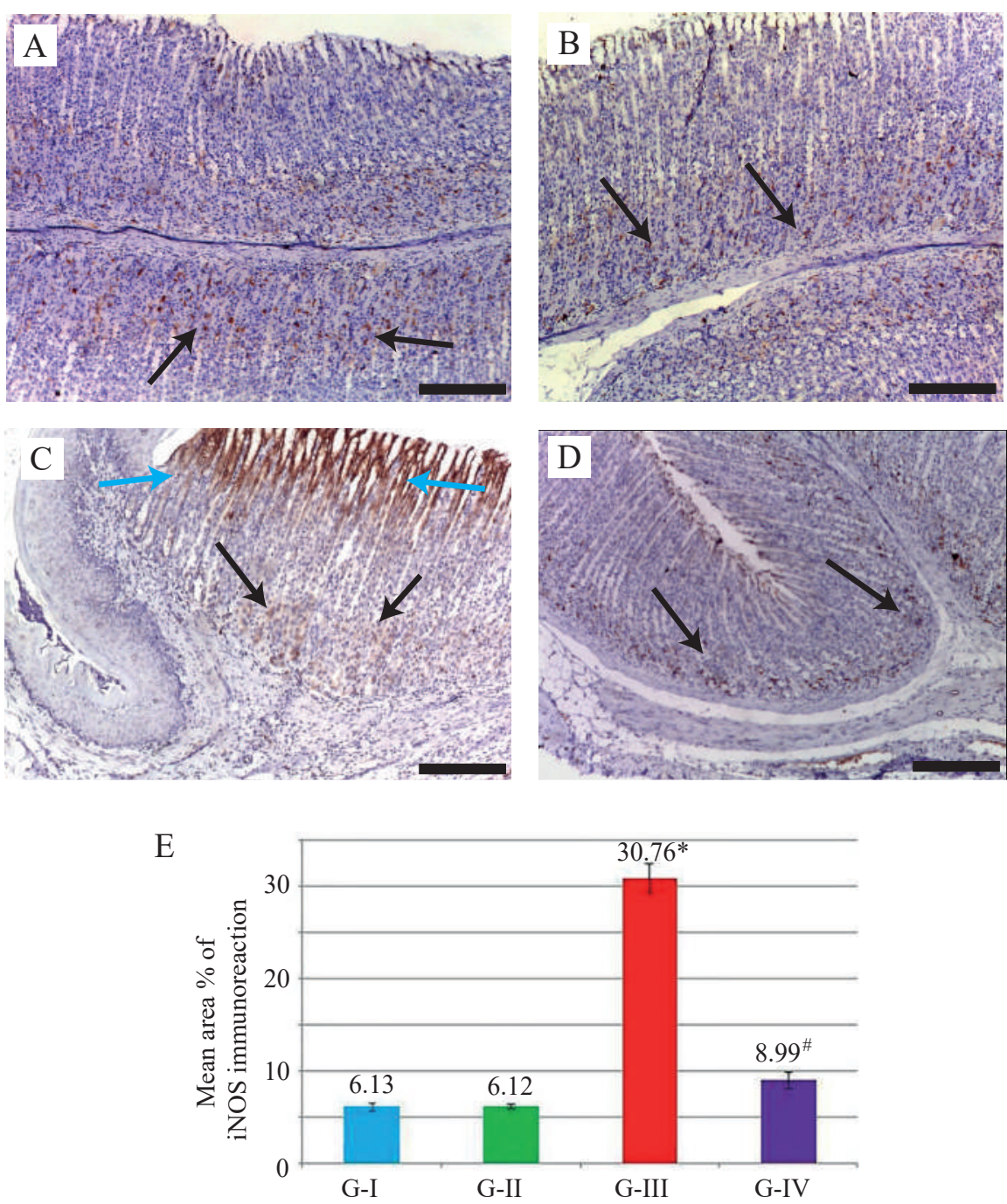

Figure 5. Photomicrographs of fundic stomach sections from different studied groups. A: G-I, showing a moderate iNOS positive reaction in the basal areas (black arrows). B: G-II, showing a moderate iNOS positive cytoplasmic reaction mainly in the basal areas (black arrows). C: G-III, showing a strong iNOS positive cytoplasmic staining mainly on the surface epithelium, gastric pits, the isthmus (blue arrows) and in the basal areas (black arrows). D: G-IV, showing a moderate iNOS cytoplasmic staining in the basal areas (black arrows). Black bar: $200 \mu \mathrm{m}$. E: Graph bars represent area percentage of iNOS reaction in the different studied groups. *Significant increase of G-III vs. G-I, while "significant decrease of G-IV vs. G-III at $p<0.001$.

Treatment with diclofenac sodium in this study resulted in the congestion of the blood vessels, degeneration of parietal cells, appearance of vacuolated cytoplasm, pyknotic nuclei, and infiltration of inflammatory cells. Similarly, earlier study using a single oral dose of $200 \mathrm{mg} /$ $\mathrm{kg}$ of aspirin produced dilatation and congestion of blood vessels.(21) Correspondingly, NSAIDs induced ischemic and inflammatory changes that lead to gastric neutrophil infiltration (22) and intestinal damage in the rat resulting in loss of the surface epithelium and numerous inflammatory cell infiltrations (23). Pyknotic nuclei could be explained due to diclofenac induced mitochondrial damage that leads to production of the reactive oxygen species resulting in apoptosis and DNA damage.(24)
Results of the present study revealed abundant collagen fibers between the disorganized fundic glands in the mucosa and around the congested blood vessels in the submucosa in the diclofenac treated group and confirmed by the morphometric study as compared with the control. Indeed, the tissue injuries were involved in fibrosis caused by chronic stimulation. Previous study suggested that the inflammatory mononuclear cells infiltrations stimulate several growth factors and attracted to the site of damage. Crucially, the basic fibroblastic growth factor enhanced fibroblast proliferation and increased collagen deposition at the site of injury and inflammation.(25)

The present study showed weak interrupted PASpositive reaction on the mucosal surface, pits and isthmus 

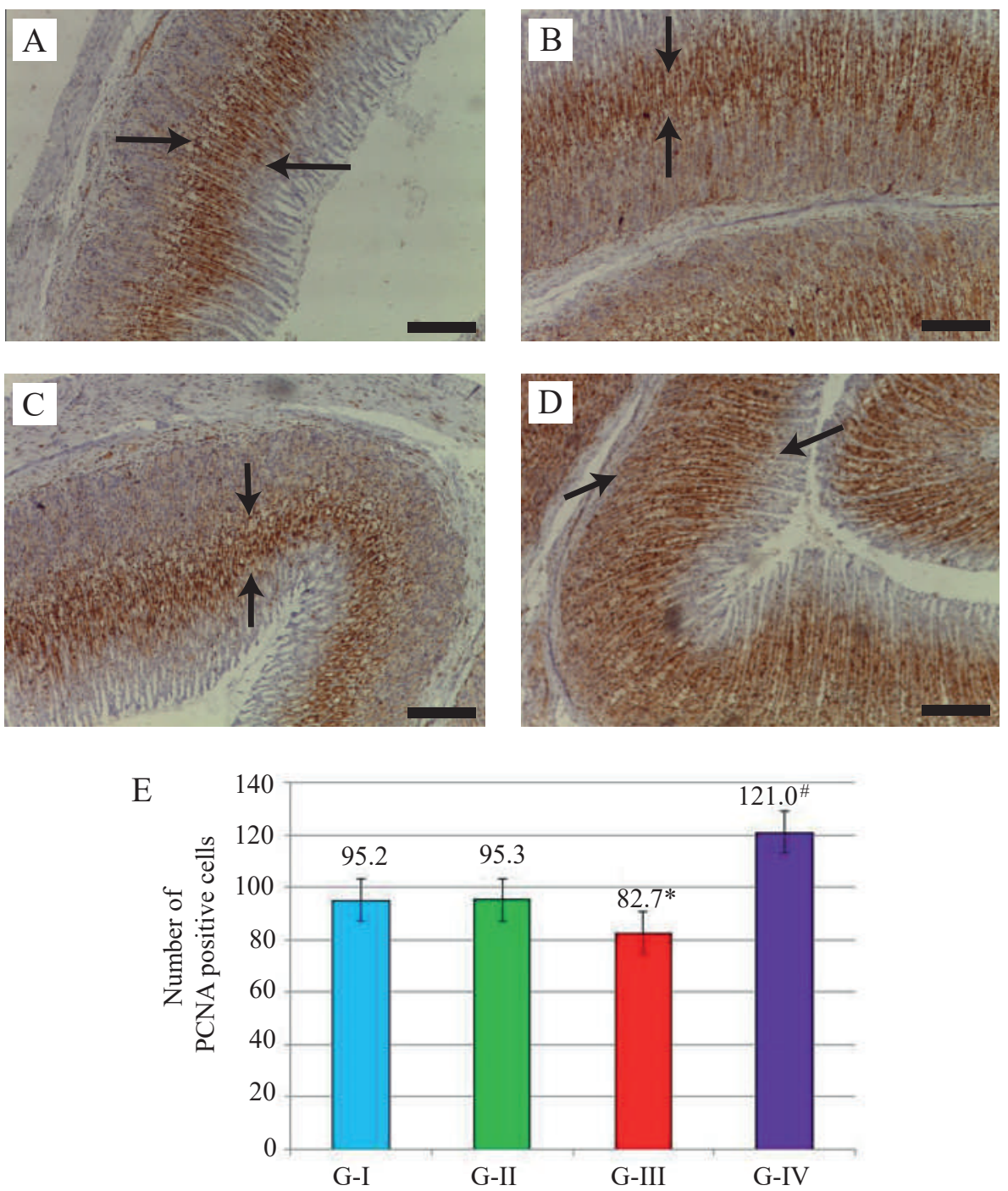

Figure 6. Photomicrographs sections of fundic gastric mucosa from different studied groups. A: G-I, showing strong positive brown PCNA immunostaining in the isthmus and neck cells (black arrows). B: G-II, showing strong positive immunoreactivity of PCNA in the isthmus and neck cells (black arrows). C: G-III, showing moderate PCNA immune reaction in the isthmus and neck cells (black arrows). D: G-IV, showing increased PCNA immune reaction in the isthmus and neck cells down to basal parts of fundic glands (black arrows). Black bar: $200 \mu \mathrm{m}$. E: Graph bars represent the number of PCNA positive cells reactions in the different studied groups. *Significant decrease of G-III vs. G-I, while "significant increase of G-IV $v s$. G-III at $p<0.001$.

in the diclofenac group and confirmed by the statistical analysis that displayed a significant decrease in the mean area percentage of the PAS positive staining as compared to control. Reduction of glycoprotein biosynthesis by the fundic epithelial cells indicated failure of gastric adaptation against the injurious effects of diclofenac sodium. Indeed, the mucous layers act as defensive factor in the stomach that protects the gastric mucosa from acid, pepsin and from bacterial infection. Mucus was decreased and disturbed due to inhibition of prostaglandin synthesis and injury of the epithelial and mucus neck cells.(26) Similarly, previous investigation suggested decrease of PAS staining during long-term administration of aspirin.(27)
The current study showed that diclofenac sodium treatment increased iNOS expression and confirmed by a significant increase in the mean area percentage of iNOS as compared to the control. It was reported that nitric oxide is essential for normal mucosal function and has a role as cytoprotective on the gastrointestinal mucosa.(28) Several investigations suggested the presence of inflammatory cellular infiltrations as lymphocytes and macrophages in the epithelium and lamina propria and up-regulation of iNOS expression during gastritis.(29) The excessive production of iNOS giving rise to inflammatory reactions that will favor the formation of gastric injuries through the generation of reactive oxygen species.(30) 

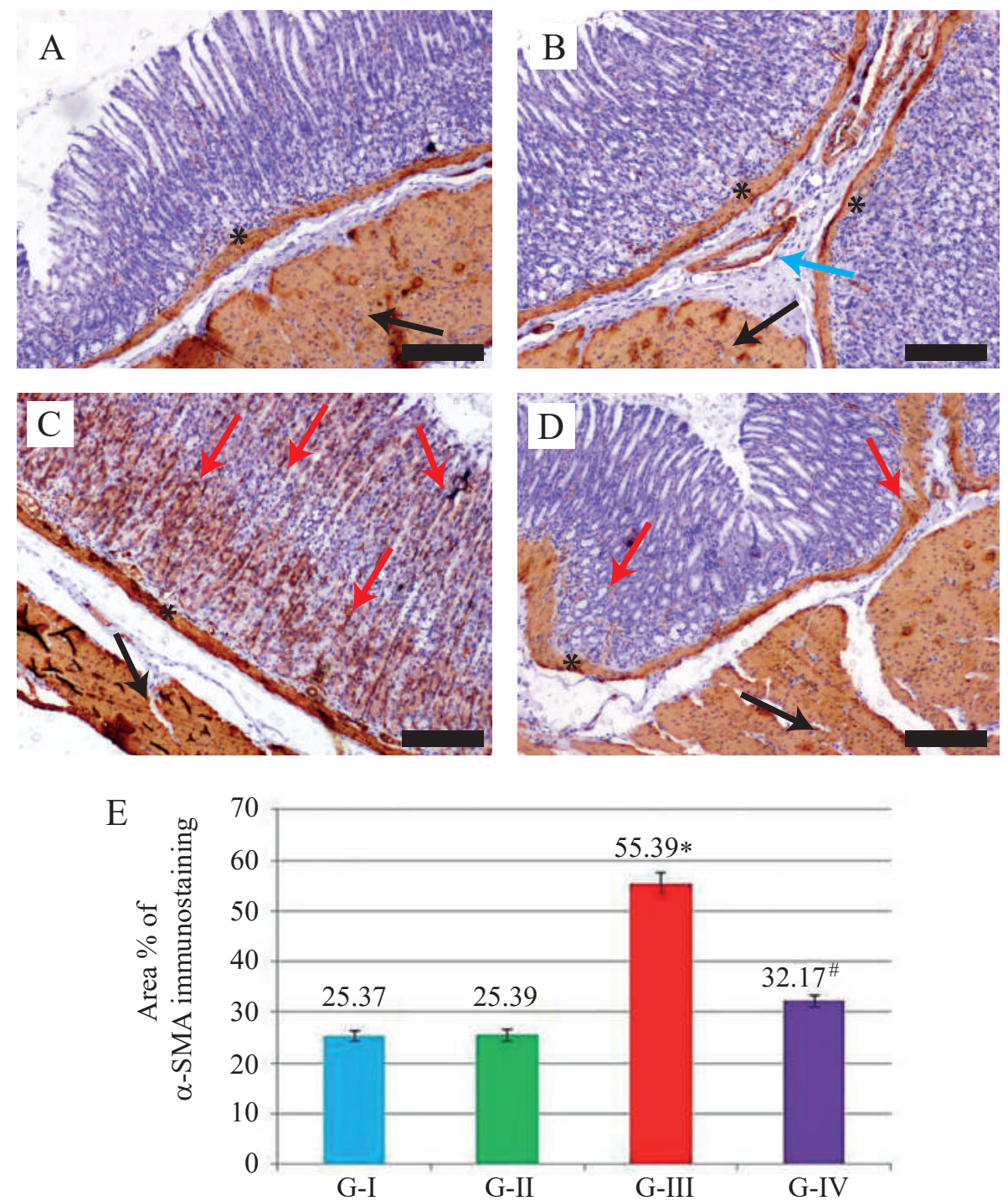

Figure 7. Photomicrographs of gastric sections from different studied groups. A: G-I, showing $\alpha$-SMA reactivity at the muscularis mucosa (star) and the muscularis externa (black arrow). B: G-II showing intense $\alpha$-SMA reactivity at the muscularis mucosa (stars) and the muscularis externa (black arrow) and around blood vessels (blue arrow). C: G-III detecting strong $\alpha$-SMA reactivity at the muscularis mucosa (star), the muscularis externa (black arrow), in the basal parts of fundic mucosal glands and focal expression in between gastric epithelium (red arrows). D: G-IV displays positive $\alpha$-SMA reactivity at the muscularis mucosa (star), the muscularis externa (black arrow) and weak staining in between the basal parts of the mucosal glands (red arrows). Black bar: $200 \mu \mathrm{m}$. E: Graph bars represent area percentage of $\alpha$-SMA detected in the different studied groups. * Significant increase of G-III vs. G-I, while *significant decrease of G-IV vs. G-III at $p<0.001$.

Proposed mechanisms of damage to the stomach include decreased cell proliferation. Cell proliferation is one of the most important roles in healing of gastric ulcers and expressed during cell proliferation. Currently, the PCNA positive immunostained cells were expressed at the isthmus and middle zone of the gastric glands in the control group. Consistently, stem cells are concentrated frequently at the middle zone. The stem cells of the gastric glands are pluripotent producing progenitor cells found within the epithelium lined the isthmus.(31) Significantly, in this work diclofenac decrease the number of mucosal PCNA positive cells. Mucosal damage might occur if the epithelial cell loss increases or decreases in the cell regeneration.

Currently, administration of diclofenac sodium increased $\alpha$-SMA expression and confirmed by a significant increase in the mean area percentage. The $\alpha$-SMA is identified in blood vessel walls and tissues which contain smooth muscle cells. Previous studied proved that expression of $\alpha$-SMA might be related to fibrotic progression. Positive link has been reported between increased $\alpha$-SMA by proliferated hepatic stellate cells, increased collagen deposition and subsequently increased grade of fibrosis.(32) 
Vitamin D, a powerful antioxidant and has been demonstrated previously to have a considerable antiinflammatory effects and might play a critical role in the immunoregulation of cytokines involved in the pathogenesis of inflammatory bowel diseases.(33) Consistently, in the present study, co-administration of vitaminD with diclofenac sodium, gastric mucosa became improved, inflammatory cells and engorged blood vessels were markedly reduced.

Presently, administration of vitamin D with diclofenac improved fundic mucosa by increasing PAS positive immunoreactions and decreasing collagen and $\alpha$ SMA. Consistently, vitamin D has been reported to improve hepatic fibrosis and diminish the secretion of collagen fibers of hepatic stellate cells. Others recommended that the combination of vitamin D and puerarin ameliorated carbon tetrachloride induced liver fibrosis of rat.(34) Moreover, vitamin $\mathrm{D}$ has high protective and therapeutic promises by increasing cell proliferation and differentiation. Currently, PCNA positive immunostained cells were markedly increased in G-IV indicating greater cell proliferation in the regenerative gastric mucosa. Importantly, the increase in the number of PCNA positive cells showed that vitamin D have a healing effect on diclofenac sodium induced gastric injury.

These results are consistent with previous reports who suggested that stem cells of gastric glands proliferate to replace all the cells lining the fundic glands. Newly cells migrate to their new position whichever deep into the gland or up into the gastric pits.(35) In the current investigation, administration of vitamin $\mathrm{D}$ with diclofenac markedly decreased iNOS positive immunoreactive cells as compared to diclofenac only. Consistently, vitamin D prevents neurotoxicity by decreasing iNOS expression.(36) Another explanation for improvements observed in G-IV was based on a potent antioxidant of vitamin D that others suggested that vitamin D supplementation prevents diabetes and cardiovascular diseases by increasing the antioxidant system such as superoxide dismutase and glutathione, however, decreasing the expression of NADPH oxidase that has the function to produce ROS.(37)

The present study has some limitations and the current data is inadequate and little is known about the associations between vitamin $\mathrm{D}$ and diclofenac sodium in gastric mucosa. It might be essential for further investigation to use vitamin $\mathrm{D}$ for a longer time for different doses or in higher or lower doses using different technique in order to determine the efficacy of optimal dose and optimal duration of vitamin D supplementation.

\section{Conclusion}

The current study suggesting the possible mechanisms involved in the gastroprotective effects of the vitamin D and its healing properties. Vitamin D is effective in the treatment of diclofenac sodium induced gastric damage by stimulating mucous secretion and decrease collagen fibers. Furthermore, its efficacy in the healing of gastric injury is based on its ability to decrease the expression of iNOS, $\alpha$-SMA and to stimulate PCNA.

\section{References}

1. Peter SJ, Basha SK, Giridharan R, Lavinya BU, Sabina EP. Suppressive effect of spirulina fusiformis on diclofenac-induced hepato-renal injury and gastrointestinal ulcer in Wistar albino rats. A biochemical and histological approach. Biomed Pharmacother. 2017; 88: 11-8.

2. Odashima M, Otaka M, Ohba R, Jin M, Wada I, Horikawa Y, et al. Attenuation of gastric mucosal inflammation induced by aspirin through inhibition of selective type III phospshodiesterase in rats. Dig Dis Sci. 2007; 52: 1355-59.

3. Watanabe T, Higuchi K, Kobata A, Nishio H, Tanigawa T. Non-steroidal antiinflammatory drug-induced small intestinal damage is Toll-like receptor 4 dependent. Gut. 2008; 57: 181-7.

4. Pastoris O, Verri M, Boschi F, Kastsiuchenka O, Balestra B, Pace F, et al. Effects of esomeprazole on glutathione levels and mitochondrial oxidative phosphorylation in the gastric mucosa of rats treated with indomethacin. Naunyn Schmied Arch Pharmacol. 2008; 378: 421-9.

5. S JP, Evan Prince S. Diclofenac-induced renal toxicity in female Wistar albino rats is protected by the pre-treatment of aqueous leaves extract of Madhuca longifolia through suppression of inflammation, oxidative stress and cytokine formation. Biomed Pharmacother. 2018; 98: 45-51.

6. Manocha S, Lal D, Venkataraman S. Administration of $\mathrm{H} 2$ blockers in NSAID induced gastropathy in rats. effect on histopathological changes in gastric, hepatic and renal tissues. Arq Gastroenterol. 2016; 53: 36-43.

7. Laine L, Takeuchi K, Tarnawski A. Gastric mucosal defense and cytoprotection: Bench to Bedside. Gastroenterol. 2008; 135: 41-60.

8. Lebda MA, El-Far AH, Noreldin AE, Elewa YHA, Al Jaouni SK, Mousa SA. Protective effects of miswak (salvadora persica) against experimentally induced Gastric Ulcers in Rats. Oxid Med Cell Longev. 2018: 2018: 6703296. doi: 10.1155/2018/6703296.

9. Tousson E, Ali EMM, Moustafa AHA, Moselhey SS, El-Said KS. Proliferating cell nuclear antigen as a biomarker for thioacetamide induced hepatotoxicity of rat liver. American Journal of Zoological Research. 2014; 2: 51-4.

10. Fuyuhiro Y, Yashiro M, Noda S, Kashiwagi S, Matsuoka J, Doi Y, et al. Myofibroblasts are associated with the progression of scirrhous gastric carcinoma. Experimental and Therapeutic Medicine. 2010; 1: $547-51$.

11. Hossein-Nezhad A, Holick MF. Vitamin D for health: a global perspective. Mayo Clin Proc. 2013; 88: 720-75.

12. Refaat B, Ahmad J, Idris S, Kamfar F, Ashshi AM, Batwa SA, Malibary FA. Characterization of vitamin D-related molecules and calcium- 
sensing receptor in human fallopian tube during the menstrual cycle and in ectopic pregnancy. Cell Tissue Res. 2017; 368: 201-13.

13. Seif AA, Abdelwahed DM. Vitamin D ameliorates hepatic ischemic/ reperfusion injury in rats. J Physiol Biochem. 2014; 70: 659-66.

14. Takayama F, Egashira T, Yamanaka Y. Effect of diclofenac, a nonsteroidal anti-inflammatory drug on lipid peroxidation caused by ischemia reperfusion in rat liver. Jpn J Pharmacol. 1994; 64: 71-8.

15. Gamble M. The hematoxylins and eosin. In: Theory and Practice of Histological Techniques. 6th ed. Bancroft JD, Gamble M, editors. Philadelphia: Elsevier; 2008. p.121-34.

16. Jones ML, Bancroft JD, Gamble M. Connective tissues and stains. In: Theory and Practice of Histological Techniques. In: Theory and Practice of Histological Techniques. 6th ed. Bancroft JD, Gamble M, editors. Philadelphia: Elsevier; 2008. p. 135-60.

17. Youssef S, Salah M. Renal cortical structural alterations in atorvastatin treated rats and the possible protective mechanisms of L-carnitine. Indian J Pharm Sci. 2019; 81: 834-43.

18. Youssef S, Mohamed SB. Impact of finasteride administration on neuroactive steroid levels to induce persistent sexual side effects and anxious/depressive disorders and the possible protective effect of vitamin E. Intl Res J Appl Basic Sci. 2017; 11: 200-20.

19. Novellino ATN, Amorim RFB, Queiroz LMG, Freitas RA. Immunoexpression analysis of PCNA and p53 in oral squamus cell carcinoma: correlation with histological grading of malignancy and clinical features. Acta Cir Bras. 2003; 18: 458-6.

20. Caselli M, Lacorte L, De Carlo L, Aleotti L, Trevsani M, Ruina F. Histological finding in gastric mucosa in patients treated with NSAIDs. J Clin Pathol. 1995; 48: 553-5.

21. Bayramli G, Ulutas B. Acute phase protein response in dogs with experimentally induced gastric mucosal injury. Vet Clin Pathol. 2008; 37: 312-16.

22. Whittle BJR. Gastrointestinal effects of nonsteroidal anti-inflammatory drugs. Fundam Clin Pharmacol. 2003; 17: 301-13.

23. Konaka A, Kato S, Tanaka A, Kunikata T, Korolkiewicz R, Takeuchi K. Roles of enterobacteria, nitric oxide and neutrophils in pathogenesis of indomethacin induced small intestinal lesions in rats. Pharmacol Res. 1999; 40: 517-24.

24. Näslund J, Fick J, Asker N, Ekman E, Larsson DGJ, Norrgren L. Diclofenac affects kidney histology in the three-spined stickleback (Gasterosteus aculeatus) at low $\mu \mathrm{g} / \mathrm{L}$ concentrations. Aquat Toxicol. 2017; 189: 87-96.

25. Kumar V, Cortan R, Robbins S. Robbins Basic Pathology. 7th ed. Philadelphia: WB Saunders Company; 2003.
26. Mohamed A. Postulated protective role of curcumin on indomethacininduced acute gastric mucosal damage in adult albino rats (Histological and Immunohistochemical Study). Egypt J Histol. 2010; 33: 583-93.

27. Gonul B, Akbulut KG, Ozer C, Yetkin G, Celebi N. The role of transforming growth factor alpha formulation on aspirin-induced ulcer healing and oxidant stress in the gastric mucosa. Surg Today. 2004; 34: 1035-40.

28. Kochar NI, Chandewal AV, Bakal RL, Kochar PN. Nitric oxide and the gastrointestinal tract. Int J Pharmacol. 2011; 7: 31-9.

29. Kai H, Ito M, Kitadai Y, Tanaka S, Haruma K, Chayama K. Chronic gastritis with expression of inducible nitric oxide synthase is associated with high expression of interleukin- 6 and hypergastrinaemia. Aliment Pharmacol Ther. 2004; 19: 1309-14.

30. de Araújo ERD, Guerra GCB, Araújo DFS, de Araújo AA, Fernandes JM, de Araújo Júnior RF da Silva VC, et al. Gastroprotective and antioxidant activity of kalanchoe brasiliensis and kalanchoe pinnata leaf juices against indomethacin and ethanol-induced gastric lesions in rats. Int J Mol Sci. 2018; 19: 1265. doi:10.3390/ ijms 19051265 .

31. Mescher AL. Junqueira's Basic Histology. Text \& Atlas. 13th ed. New York: McGraw Hill; 2013.

32. Hashem MM, Salama MM, Mohammed FF, Tohamy AF, El Deeb KS. Metabolic profile and hepatoprotective effect of Aeschynomene elaphroxylon (Guill. \& Perr.). PLoS ONE. 2019; 14(1): e0210576. doi: 10.1371/ journal. pone.0210576.

33. Choi M, Park H, Cho S, Lee M. Vitamin D3 supplementation modulates inflammatory responses from the muscle damage induced by highintensity exercise in SD rats. Cytokine. 2013; 63: 27-35.

34. Huang GR, Wei SJ, Huang YQ, Xing W, Wang LY, Liang LL. Mechanism of combined use of vitamin D and puerarin in antihepatic fibrosis by regulating the $\mathrm{Wnt} / \beta$-catenin signalling pathway. World J Gastroenterol. 2018; 28; 24: 4178-85.

35. Gartner LP, Hiatt JL. Color Textbook of Histology. 2nd ed. Philadelphia: WB. Saunders Company; 2001.

36. Dursun E, Gezen-AKD, Yilmazer S. The influence of vitamin D treatment on the inducible nitric oxide synthase (INOS) expression in primary hippocampal neurons. Noro Psikiyatr Ars. 2014; 51: 163-8.

37. El-Gohary OA, Allam MM. Effect of vitamin D on isoprenalineinduced myocardial infarction in rats: possible role of peroxisome proliferator-activated receptor- $\gamma$. Can J Physiol Pharmacol. 2017; 95: 641-6. 